\title{
Capacity Analysis of MIMO Three Product Channels
}

\author{
Abdulla Firag $^{\dagger} \quad$ Peter J. Smith ${ }^{\ddagger} \quad$ Matthew R. McKay ${ }^{\dagger \dagger}$ \\ $\dagger$ Dept. of Mathematics and Statistics, University of Canterbury, Christchurch, New Zealand \\ $\ddagger$ Dept. of Electrical and Computer Engineering and Mathematics and Statistics \\ University of Canterbury, Christchurch, New Zealand \\ $\dagger^{\dagger}$ Dept. of Electronic and Computer Engineering \\ Hong Kong University of Science and Technology, Hong Kong
}

\begin{abstract}
The paper presents an ergodic capacity analysis of MIMO three product channels. We first derive the exact non-asymptotic and asymptotic probability density functions of the arbitrary eigenvalue of the system. From these, a nonasymptotic and asymptotic expression for the ergodic capacity of the system is derived. The non-asymptotic results involve several single numerical integrations and the number of numerical integrations increases with the number of antennas used in the system. However, the asymptotic result has only one numerical integration and a more compact form, hence providing a simpler alternative to the non-asymptotic ergodic capacity result. We also validate the results by using simulations. The results show that the non-asymptotic analytical results are in good agreement with the simulations and the asymptotic analytical results provide a good approximation to the exact results even when the system has very few antennas.
\end{abstract}

\section{INTRODUCTION}

Multiple-input, multiple-output (MIMO) product channels have recently been given considerable attention due to their many applications. Wireless channels are modeled as MIMO product channels in MIMO multi-keyhole environments [1], [2], [3] and also in certain MIMO multi-hop relaying environments [4], [5], [6], [7]. In most of this work performance analysis has focused on two product channels of finite size. In addition capacity results on MIMO two product channels have also been derived by employing asymptotic methods [1], [8], [9], [10]. The advantage of the asymptotic methods is their ability to provide accurate results with greatly reduced complexity.

In this paper, we analyze the ergodic capacity of MIMO three product channels. The exact analysis builds on the approach in [6] while the large system approach requires an asymptotic eigenvalue analysis of MIMO product channels. In [11], [12], the arbitrary eigenvalue probability density function of a Wishart matrix is studied as the dimension of the matrix becomes large. In [1] a similar study is performed for MIMO two product channels. Here, the author uses the Stransform and the Stieltjes transform to derive an asymptotic arbitrary eigenvalue probability density function (p.d.f.) for the MIMO two product channel. The Stieltjes transform has recently been identified as a key tool to derive information- and communication-theoretic performance measures for random vector channels [1]. A general method to obtain the Stieltjes transform for MIMO $N$ product channels is discussed in [13]. Furthermore, in [14] an asymptotic capacity analysis is performed for multi-hop relaying systems again using the Stieltjes transform. In this work a numerical method is used to the obtain asymptotic arbitrary eigenvalue distribution from the Stieltjes transform and no closed form expression for the capacity is given. However, asymptotic ergodic capacity analysis for MIMO three product channels is not accurately available in the literature. Hence, our main contribution in this paper is to derive an exact expression for the ergodic capacity of MIMO three product channels and also to derive an asymptotic expression for the ergodic capacity of the channels using the Stieltjes transform. Our asymptotic result is much simpler than the exact expression and provides a very good approximation even when the number of antennas is very small. We also present simulation results to validate our analysis.

\section{System MODEL}

MIMO product channels arise in MIMO environments when there are groups of scattering objects [1] and are also found in MIMO multi-hop relaying environments [3]. Specifically, MIMO three product channels can be found in an environment where there are two clusters of scatterers in between the source and destination terminals. One such environment is the propagation of signals between different floors of a building [13]. Furthermore, MIMO three product channels can also be found in MIMO 3-hop relaying environments when the amplified noise from the relays is negligible. For ease of exposition we present our analysis in the context of the MIMO threehop relaying system shown in Fig. 1. The source $(S)$, relays $\left(R_{i}\right)$, and destination $(D)$ terminals are equipped with $n_{s}$, $n_{r}$ and $n_{d}$ antennas respectively. We assume the relays have equal numbers of antennas due to space limitations. When the relays have arbitrary numbers of antennas, the analysis is still possible but additional cases have to be considered separately. During the first hop, $S$ transmits to $R_{1}$, in the second hop $R_{1}$ transmits the amplified signal from the first hop to $R_{2}$ and finally in the third hop $R_{2}$ transmits the amplified signal from the second hop to $D$. We let the normalized channel matrices 


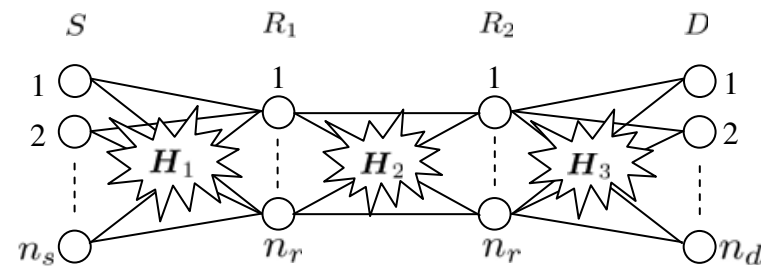

Fig. 1. MIMO three-hop relaying system.

for the source-to-relay $\left(S \rightarrow R_{1}\right)$, relay-to-relay $\left(R_{1} \rightarrow R_{2}\right)$, and relay-to-destination $\left(R_{2} \rightarrow D\right)$ links be given by $\boldsymbol{H}_{1} \in \mathcal{C}^{n_{r} \times n_{s}}$, $\boldsymbol{H}_{2} \in \mathcal{C}^{n_{r} \times n_{r}}$, and $\boldsymbol{H}_{3} \in \mathcal{C}^{n_{d} \times n_{r}}$, respectively, and assume that both relays have $n_{r}$ antennas. All channels are assumed to exhibit independent and identically-distributed (i.i.d.) flat Rayleigh fading. Hence, the entries of the corresponding channel matrices are modeled as i.i.d. zero mean circularly symmetric complex Gaussian (ZMCSCG) random variables with unit variance. Furthermore, we assume that relays assist in the communication with $D$ using amplify-and-forward (AF) relaying. Hence, $R_{i}$ amplifies the received observation by a factor, $b_{i}$, and retransmits it to the corresponding destination. The received signal at the destination after the three hops is then given by

$$
\begin{aligned}
\boldsymbol{y} & =b_{2} b_{1} \sqrt{P_{3} P_{2} P_{1}} \boldsymbol{H}_{3} \boldsymbol{H}_{2} \boldsymbol{H}_{1} \boldsymbol{x} \\
& +b_{2} b_{1} \sqrt{P_{3} P_{2}} \boldsymbol{H}_{3} \boldsymbol{H}_{2} \boldsymbol{n}_{1}+b_{2} \sqrt{P_{3}} \boldsymbol{H}_{3} \boldsymbol{n}_{2}+\boldsymbol{n}_{3} .
\end{aligned}
$$

In (1), the parameters $P_{1}, P_{2}$ and $P_{3}$ are the average powers of the $S \rightarrow R_{1}, R_{1} \rightarrow R_{2}$ and $R_{2} \rightarrow D$ links, respectively, taking into account the different path loss and shadowing effects over the links. The variables $\boldsymbol{n}_{1}, \boldsymbol{n}_{2}$ and $\boldsymbol{n}_{3}$ are the noise vectors at $R_{1}, R_{2}$ and $D$ respectively, and $\boldsymbol{x}$ is the vector of transmit symbols. The transmit symbols are assumed i.i.d. with $E\left\{\boldsymbol{x} \boldsymbol{x}^{\dagger}\right\}=\rho_{x} \boldsymbol{I}_{n_{s}} / n_{s}$. The noise at $D$ is modeled as ZMCSCG with $E\left\{\boldsymbol{n}_{3} \boldsymbol{n}_{3}^{\dagger}\right\}=\boldsymbol{I}_{n_{d}}$. To obtain the 3 product channel we follow [3] and assume that the amplified noises from the relays are negligible. With this assumption, the received signal at the destination can be written as

$$
\begin{aligned}
\boldsymbol{y} & =b_{2} b_{1} \sqrt{P_{3} P_{2} P_{1}} \boldsymbol{H}_{3} \boldsymbol{H}_{2} \boldsymbol{H}_{1} \boldsymbol{x}+\boldsymbol{n}_{3} \\
& \triangleq \sqrt{\rho_{1}} \boldsymbol{H}_{3} \boldsymbol{H}_{2} \boldsymbol{H}_{1} \boldsymbol{x}+\boldsymbol{n}_{3},
\end{aligned}
$$

where $\rho_{1}=b_{2}^{2} b_{1}^{2} P_{3} P_{2} P_{1}$. In this paper, we discuss the ergodic capacity of the received signal given in (2) both asymptotically and non-asymptotically.

\section{NON-ASYMPTOTIC CAPACITY ANALYSIS}

Defining $\rho \triangleq \rho_{1} \rho_{x}$, the ergodic capacity of the system is given by [5] as below,

$$
C=\frac{1}{3} E\left\{\log _{2}\left|\boldsymbol{I}_{n_{d}}+\frac{\rho}{n_{s}} \boldsymbol{H}_{3} \boldsymbol{H}_{2} \boldsymbol{H}_{1}\left(\boldsymbol{H}_{3} \boldsymbol{H}_{2} \boldsymbol{H}_{1}\right)^{\dagger}\right|\right\} .
$$

The factor $1 / 3$ accounts for the fact that information is conveyed to the destination terminal over three time slots [4]. This factor can be increased by pipelining the transmission of messages. However, it does not effect the analysis and hence we assumed that no pipelining is used in the transmission.
For the capacity analysis, we require the distribution of the non-zero arbitrary eigenvalue, $\lambda$, of $\boldsymbol{W}$ where

$$
\boldsymbol{W}=\boldsymbol{H}_{3} \boldsymbol{H}_{2} \boldsymbol{H}_{1}\left(\boldsymbol{H}_{3} \boldsymbol{H}_{2} \boldsymbol{H}_{1}\right)^{\dagger} \text {. }
$$

However, a general form for this distribution is not possible for arbitrary numbers of antennas. Thus, we have to consider two separate cases: $n_{r} \leqslant n_{s}$ and $n_{r}>n_{s}$. Let $s_{0}=\min \left(n_{d}, n_{r}\right)$, $s_{1}=\min \left(n_{d}, n_{s}\right)$ and $s=\min \left(s_{0}, s_{1}\right)$, then the ergodic capacity can also be written as

$$
C=\frac{s}{3 \ln (2)} \int_{0}^{\infty} \ln \left(1+\rho \lambda / n_{s}\right) f(\lambda) d \lambda,
$$

where $f(\lambda)$ is the probability density function (p.d.f.) of $\lambda$. Hence, to find the ergodic capacity of the system, we need to find the non-zero arbitrary eigenvalue density, $f(\lambda)$, of the random matrix $\boldsymbol{W}$. The derivations of the ergodic capacity of the system for the two cases are given below.

\section{A. Capacity Analysis: $n_{r} \leqslant n_{s}$}

For this case the non-zero arbitrary eigenvalue density, $f(\lambda)$, can be given as follows.

Theorem 1: The p.d.f. of an arbitrary eigenvalue $\lambda$ of $\boldsymbol{W}$ when $n_{r} \leqslant n_{s}$ is given by

$$
\begin{aligned}
f(\lambda) & =\frac{1}{\Gamma_{n_{r}}\left(n_{r}\right) \Gamma_{n_{r}}\left(n_{r}\right) \Gamma_{n_{r}}\left(n_{s}\right) s_{0}} \\
& \times \sum_{i=n_{r}-s_{0}+1}^{n_{r}} \sum_{j=1}^{n_{r}}(-1)^{i+j} \frac{\lambda^{n_{d}-n_{r}+i-1}}{\Gamma\left(n_{d}-n_{r}+i\right)}\left|\boldsymbol{K}_{i, j}\right| A_{\lambda}(j),
\end{aligned}
$$

where $\Gamma_{m}(n)=\prod_{k=1}^{m} \Gamma(n-k+1), \boldsymbol{K}_{i, j}$ denotes the $(i, j)^{\text {th }}$ minor of the $n_{r} \times n_{r}$ matrix $\boldsymbol{K}$ with elements

$$
\boldsymbol{K}_{i, j}=\Gamma(i+j-1) \Gamma\left(n_{s}-n_{r}+i\right),
$$

and

$$
A_{\lambda}(j)=\int_{0}^{\infty} 4 u^{\left(n_{s}+n_{r}-2 n_{d}+j-2\right)} e^{-\frac{\lambda}{u^{2}}} K_{n_{r}-n_{s}+j-1}(2 u) d u .
$$

In (8), $K_{x}(\cdot)$ is the modified Bessel function of the second kind.

Proof: See Appendix A.

Then, using the above result and (5) and defining $\rho_{0}=$ $\rho / n_{s}$, the ergodic capacity can be calculated as

$$
\begin{aligned}
C= & \frac{1}{3 \ln (2) \Gamma_{n_{r}}\left(n_{r}\right) \Gamma_{n_{r}}\left(n_{r}\right) \Gamma_{n_{r}}\left(n_{s}\right)} \sum_{i=n_{r}-s_{0}+1}^{n_{r}} \sum_{j=1}^{n_{r}}(-1)^{i+j} \\
& \times\left|\boldsymbol{K}_{i, j}\right| \underbrace{\int_{0}^{\infty} \ln \left(1+\rho_{0} \lambda\right) \frac{\lambda^{n_{d}-n_{r}+i-1}}{\Gamma\left(n_{d}-n_{r}+i\right)} A_{\lambda}(j) d \lambda}_{I_{B}}, \text { (9) }
\end{aligned}
$$

where $I_{B}$ can be evaluated as

$$
\begin{aligned}
I_{B}= & \sum_{k=0}^{r} 4 k !\left(\begin{array}{c}
r \\
k
\end{array}\right) \rho^{k-r} \frac{\Gamma(r-k+1)}{\Gamma\left(n_{d}-n_{r}+i\right)} \\
& \times \int_{0}^{\infty} u^{\left(n_{s}+n_{r}+2 k-2 n_{d}+j\right)} e^{\frac{1}{\rho u^{2}}} \Gamma\left(k-r, 1 /\left(\rho u^{2}\right)\right) \\
& \times K_{n_{r}-n_{s}+j-1}(2 u) d u
\end{aligned}
$$


where $r=n_{d}-n_{r}+i-1$. A closed form expression for the integral, $I_{B}$, in (10) is difficult to obtain and numerical integration is required.

\section{B. Capacity Analysis: $n_{r}>n_{s}$}

For this case the non-zero arbitrary eigenvalue density, $f(\lambda)$, can be given as follows.

Theorem 2: The p.d.f. of an arbitrary eigenvalue $\lambda$ of $\boldsymbol{W}$ when $n_{r}>n_{s}$ is given by

$$
\begin{aligned}
& f(\lambda)=\frac{1}{s_{1} \Gamma_{n_{s}}\left(n_{s}\right) \Gamma_{n_{r}}\left(n_{r}\right) \Gamma_{n_{r}}\left(n_{r}\right)} \sum_{i=n_{s}-s_{1}+1}^{n_{s}} \sum_{j=1}^{n_{r}} \\
& \quad \times(-1)^{n_{r}-n_{s}+i+j} \frac{\lambda^{n_{d}-n_{s}+i-1}}{\Gamma\left(n_{d}-n_{s}+i\right)}\left|\boldsymbol{K}_{n_{r}-n_{s}+i, j}^{p s}\right| A_{\lambda}(j)
\end{aligned}
$$

where $\boldsymbol{K}_{i, j}^{p s}$ denotes the $(i, j)^{\mathrm{th}}$ minor of the $n_{r} \times n_{r}$ matrix $\boldsymbol{K}^{p s}$ with elements

$$
\boldsymbol{K}_{i, j}^{p s}= \begin{cases}\Gamma(i+j-1) & i=1, \ldots, n_{r}-n_{s} \\ \Gamma(i+j-1) & i=n_{r}-n_{s}+1, \ldots, n_{r} \\ \times \Gamma\left(n_{s}-n_{r}+i\right) & \end{cases}
$$

and $A_{\lambda}(j)$ is given in (8).

Proof: See Appendix B.

Using the results in (11) and (5), the ergodic capacity for $n_{r}>n_{s}$ can be calculated as

$$
\begin{aligned}
C= & \frac{1}{3 \ln (2) \Gamma_{n_{r}}\left(n_{r}\right) \Gamma_{n_{r}}\left(n_{r}\right) \Gamma_{n_{s}}\left(n_{s}\right)} \sum_{i=n_{s}-s_{1}+1}^{n_{s}} \sum_{j=1}^{n_{r}} \\
& \times(-1)^{n_{r}-n_{s}+i+j}\left|\boldsymbol{K}_{n_{r}-n_{s}+i, j}^{p s}\right| \\
& \times \underbrace{\int_{0}^{\infty} \ln \left(1+\rho_{0} \lambda\right) \frac{\lambda^{n_{d}-n_{s}+i-1}}{\Gamma\left(n_{d}-n_{s}+i\right)} A_{\lambda}(j) d \lambda}_{I_{B}^{p s}},
\end{aligned}
$$

where $I_{B}^{p s}$ can be evaluated as

$$
\begin{aligned}
I_{B}^{p s}= & \sum_{k=0}^{r_{1}} 4 k !\left(\begin{array}{c}
r_{1} \\
k
\end{array}\right) \rho^{k-r_{1}} \frac{\Gamma\left(r_{1}-k+1\right)}{\Gamma\left(n_{d}-n_{s}+i\right)} \\
& \times \int_{0}^{\infty} u^{\left(n_{s}+n_{r}+2 k-2 n_{d}+j\right)} e^{\frac{1}{\rho u^{2}}} \Gamma\left(k-r_{1}, 1 /\left(\rho u^{2}\right)\right) \\
& \times K_{n_{r}-n_{s}+j-1}(2 u) d u
\end{aligned}
$$

where $r_{1}=n_{d}-n_{s}+i-1$. Again, a closed form expression for the integral, $I_{B}^{p s}$, in (14) is difficult to obtain and a single numerical integration is required.

\section{Asymptotic CAPACity ANALYsis}

The non-asymptotic capacity results in the previous section gives exact capacity results for any number of antennas. However, the non-asymptotic results involve numerical integrations and determinants. Furthermore, the number of numerical integrations and determinants that need to be computed increases with an increase in the number of antennas in the system. In this section, we derive an asymptotic capacity result that serves as a good estimate for the non-asymptotic case with greatly reduced complexity.

The asymptotic capacity analysis considers the arbitrary eigenvalue p.d.f. of $\boldsymbol{W}$ given in (4) when $n_{s} / n_{r} \rightarrow c_{0}$ and $n_{d} / n_{r} \rightarrow c_{1}$ as $n_{s}, n_{r}, n_{d} \rightarrow \infty$ for positive constants $c_{0}$, $c_{1}$. In [1] it is shown that the Stieltjes transform can be used to find the asymptotic arbitrary eigenvalue distribution of MIMO product channels. The Stieltjes transform is related to the asymptotic p.d.f. of an arbitrary eigenvalue, $f_{a}(\lambda)$, of a random matrix as

$$
G(s)=\int_{-\infty}^{\infty} \frac{f_{a}(\lambda)}{\lambda+s} d \lambda .
$$

In [13], the author derives a general method to obtain the Stieltjes transform for matrices of type

$$
\boldsymbol{C}_{N}=\boldsymbol{H}_{1} \ldots \boldsymbol{H}_{N}\left(\boldsymbol{H}_{1} \ldots \boldsymbol{H}_{N}\right)^{\dagger} /\left(n^{N}\right),
$$

where $\boldsymbol{H}_{i}$ is $n \times n$ matrix with entries which are i.i.d. ZMCSCG random variables with unit variance. Using the result in [13], we can obtain the Stieltjes transform of $\boldsymbol{W}$ for the general case of arbitrary values of $c_{0}, c_{1}$. However, the general result is complex and due to space limitations we assume $n_{s}=n_{r}=n_{d} \triangleq n$ in the derivation below. Hence, using the result in [13] and assuming $n_{s}=n_{r}=n_{d} \triangleq n$, the Stieltjes transform of $\boldsymbol{W} / n^{3}$ can be given as,

$$
s^{3} G^{4}(s)+s G(s)-1=0 .
$$

The asymptotic p.d.f. of an arbitrary eigenvalue of the random matrix $\boldsymbol{W} / n^{3}, f_{a}(\lambda)$, can be obtained using the Stieltjes inversion formula [1]

$$
f_{a}(\lambda)=\frac{1}{\pi} \lim _{\epsilon \rightarrow 0} \Im G(-\lambda-j \epsilon),
$$

where $\Im Z$ is the imaginary part of $Z$. Using (18), $f_{a}(\lambda)$ can be obtained as below.

Let

$$
\begin{aligned}
& g(\lambda)=\lim _{\epsilon \rightarrow 0} \Re G(-\lambda-j \epsilon), \\
& c(\lambda)=\lim _{\epsilon \rightarrow 0} \Im G(-\lambda-j \epsilon),
\end{aligned}
$$

where $\Re Z$ is the real part of $Z$. For $c(\lambda) \neq 0$, the real and imaginary parts of (17) give the following system of equations:

$$
\begin{gathered}
\lambda^{3} g^{4}(\lambda)-6 \lambda^{3} c^{2}(\lambda) g^{2}(\lambda)+\lambda^{3} c^{4}(\lambda)+\lambda g(\lambda)+1=0 \\
4 \lambda^{2} g^{3}(\lambda)-4 \lambda^{2} c^{2}(\lambda) g(\lambda)+1=0
\end{gathered}
$$

respectively. Solving (22) for $c^{2}(\lambda)$ gives

$$
c^{2}(\lambda)=g^{2}(\lambda)+\frac{1}{4 \lambda^{2} g(\lambda)} .
$$

Solving (21) for $c^{2}(\lambda)$ and setting its solution equal to the right-hand side of (23) gives

$$
64 \lambda^{4} g^{6}(\lambda)-16 \lambda g^{2}(\lambda)-1=0 .
$$

As $c(\lambda)$ and $f_{a}(\lambda)$ are related by $f_{a}(\lambda)=c(\lambda) / \pi, c^{2}(\lambda)$ has to be strictly non-negative and real in the correct domain. From 
(23), this also means that $g^{2}(\lambda)$ has to be strictly positive and real in the correct domain. The random matrix $\boldsymbol{W} / n^{3}$ is positive-definite when $n_{s}=n_{r}=n_{d}$. Hence, $f_{a}(\lambda)$ will be zero when $\lambda \leqslant 0$, positive and continuous in the range, $0<\lambda<\lambda_{\max }$ and zero when $\lambda \geqslant \lambda_{\max }$. Now using this information, equation (24) and the properties of cubic polynomials, it can be found that $g^{2}(\lambda)$ has real and positive values when $0<\lambda<16^{2} / 27$ and $g^{2}(\lambda)$ satisfying these conditions can be given as

$$
\begin{gathered}
g^{2}(\lambda)=\frac{\sqrt[3]{64^{2} \lambda^{8}}(1-j \sqrt{3})}{384 \lambda^{4}} \sqrt[3]{\frac{-27+\sqrt{27^{2}-27 \times 16^{2} / \lambda}}{2}} \\
+\frac{\sqrt[3]{64^{2} \lambda^{8}}(1+j \sqrt{3})}{384 \lambda^{4}} \sqrt[3]{\frac{-27-\sqrt{27^{2}-27 \times 16^{2} / \lambda}}{2}}
\end{gathered}
$$

From the properties of $f_{a}(\lambda), c\left(\lambda_{\max }\right)$ must be equal to 0 . Hence, observing (23) and (25), $\lambda_{\max }$ must be equal to $16^{2} / 27$. Also using (23), $c\left(\lambda_{\max }\right)=0$ only when $g(\lambda)$ has negative values. Hence, $g(\lambda)$ has to be real and negative. Thus, $g(\lambda)=-\sqrt{g^{2}(\lambda)}$, where $g^{2}(\lambda)$ is given in (25).

Now, using the above information, $f_{a}(\lambda)$ can be calculated as

$$
f_{a}(\lambda)=\frac{1}{\pi} \sqrt{g^{2}(\lambda)+\frac{1}{4 \lambda^{2} g(\lambda)}},
$$

where $g(\lambda)$ is given above and $f_{a}(\lambda)$ is defined in $0<\lambda<$ $16^{2} / 27$ and is zero elsewhere. Using this result, the asymptotic ergodic capacity for the system defined in (2) can be written as

$$
C_{a}=\frac{n}{3 \ln (2)} \int_{0}^{16^{2} / 27} \ln \left(1+n^{2} \rho \lambda\right) f_{a}(\lambda) d \lambda
$$

where we have assumed $n_{s}=n_{r}=n_{d} \triangleq n$. From (27), the asymptotic ergodic capacity result has only one numerical integration and the integrand is a single closed form expression. Hence, the asymptotic result is much simpler than the non-asymptotic ergodic capacity result given in (9).

It is possible to obtain $f_{a}(\lambda)$ for the general case when $n_{s}, n_{r}$ and $n_{d}$ are unequal and $c_{0}, c_{1}$ are arbitrary positive numbers. In this case, the sixth order polynomial equation in $g(\lambda),(24)$, has non vanishing terms in all powers of $g(\lambda)$ up to order 6 . Hence, the solution for $g(\lambda)$ does not have a simple closed form as in (25). Therefore, the solution for $g(\lambda)$ has to be evaluated numerically and the correct root out, of the 6 possibilities, has to be identified. In this case, $f_{a}(\lambda)$ also includes zero eigenvalues. Hence, $f_{a}(\lambda)$ has to be scaled by a factor, $n_{d} / \min \left(n_{s}, n_{r}, n_{d}\right)$, so that the arbitrary eigenvalue p.d.f. obtained only includes non-zero eigenvalues. We include some results for this case in Sec. V but a full exposition of the calculations is omitted for reason of space.

\section{REsUlts}

The results produced in this paper are validated by using Monte Carlo simulation. In all the results given, we have used

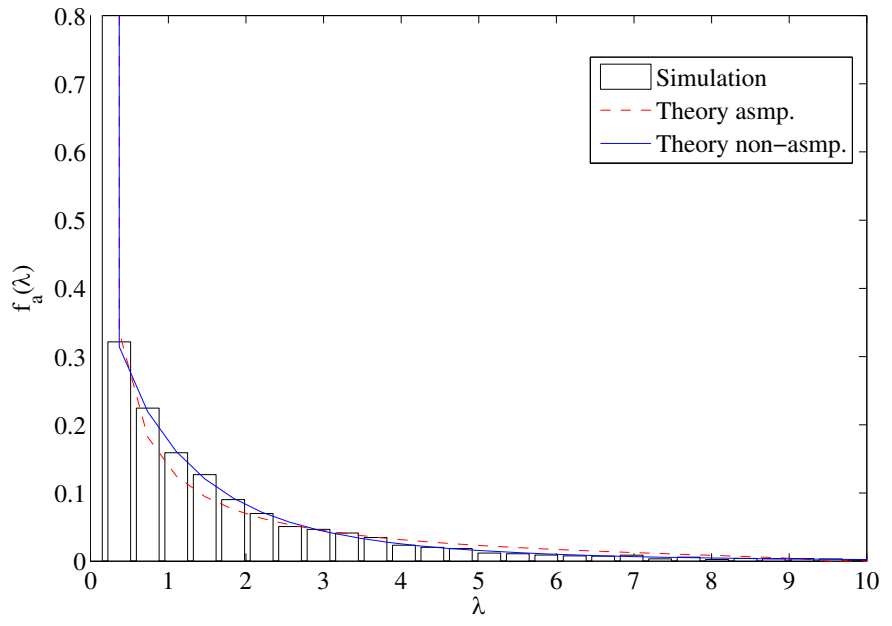

Fig. 2. Analytical and simulated p.d.f.s of the arbitrary eigenvalue of $\boldsymbol{W} / n^{3}$, with system parameters: $(2,2,2)$.

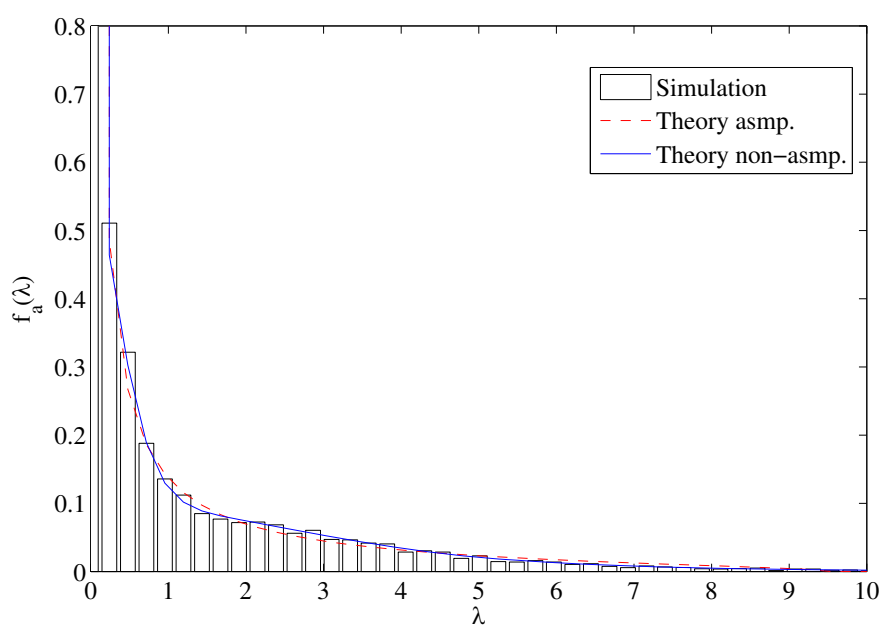

Fig. 3. Analytical and simulated p.d.f.s of the arbitrary eigenvalue of $\boldsymbol{W} / n^{3}$, with system parameters: $(4,4,4)$.

the system defined in (2) and the number of antennas used in the system is represented by the 3-tuple $\left(n_{s}, n_{r}, n_{d}\right)$. First, in Figs. 2 and 3, we validate the result in Theorem 1 and the asymptotic p.d.f., $f_{a}(\lambda)$, via simulation. The result in Theorem 1 is for an arbitrary eigenvalue p.d.f. of $\boldsymbol{W}$. Hence, it needs to be scaled such that $n^{3} f\left(n^{3} \lambda\right)$ will give the p.d.f. of the arbitrary eigenvalue of $\boldsymbol{W} / n^{3}$. The plots show the p.d.f. of the arbitrary eigenvalue, $\lambda$, with system configurations $(2,2,2)$ and $(4,4,4)$. Figure 2 shows that the non-asymptotic analytical results are in good agreement with the simulations but the asymptotic analytical results have some minor differences compared to the simulations. However, Fig. 3 shows that this difference is smaller for larger systems.

Figure 4 gives the non-asymptotic, asymptotic and simulated ergodic capacity per antenna of the system defined in (2). The non-asymptotic and asymptotic analytical results are based on (9) and (27), respectively, and the results are given for the system configurations: $(2,2,2),(4,4,4)$ and $(6,6,6)$. 


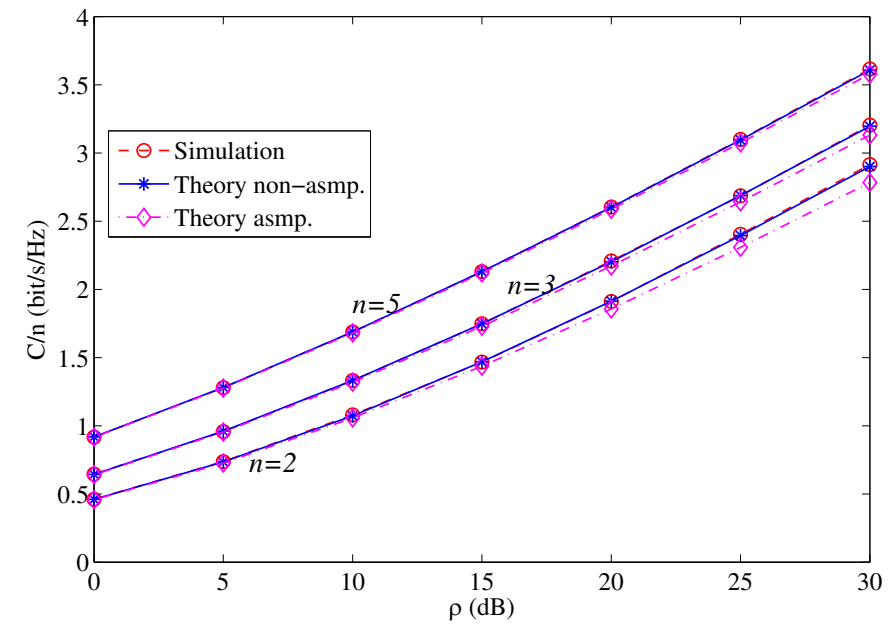

Fig. 4. Analytical and simulated ergodic capacity per antenna of the system with parameters: $(2,2,2),(3,3,3),(5,5,5)$.

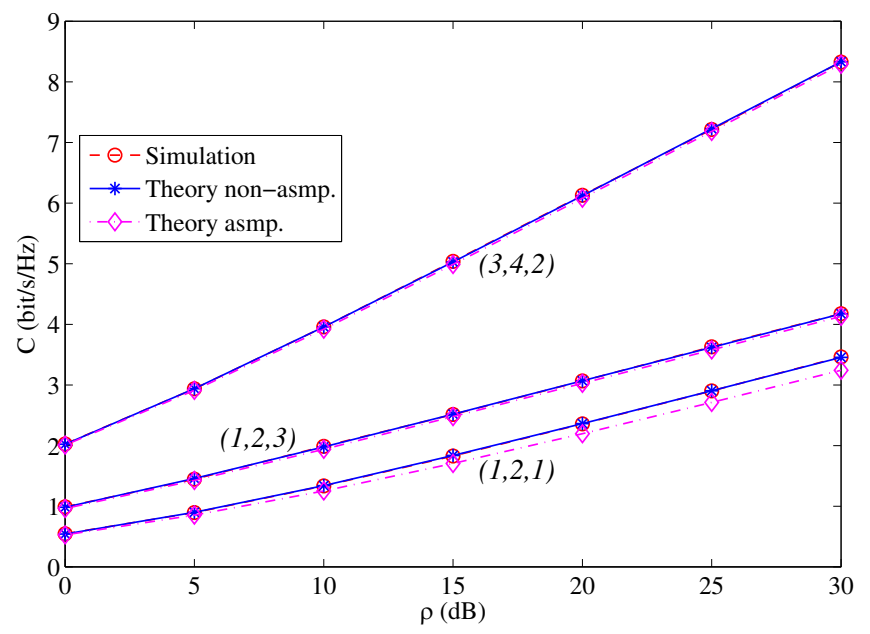

Fig. 5. Analytical and simulated ergodic capacity of the system with parameters: $(1,2,3),(3,4,2),(1,2,1)$.

Figure 4 shows that the non-asymptotic analytical results are in good agreement with the simulations for all values of $n$ and the asymptotic analytical results have minor differences compared with the simulations in the higher SNR regions and when $n$ is small. However, the asymptotic analytical results improve as $n$ increases and give a good estimate for the nonasymptotic results for $n$ as low as 3 .

Finally, we validate the analytical ergodic capacity result given in Sec. III for the case when $n_{r}>n_{s}$ by using simulation. Here, we also include results for the general case when $n_{s}, n_{r}$ and $n_{d}$ are unequal. Figure 5 shows that the nonasymptotic analytical results are in good agreement with the simulations and the asymptotic results provide an excellent approximation to the exact results.

\section{CONClusions}

The paper presents an ergodic capacity analysis of MIMO three product channels. We first derived the exact non- asymptotic and asymptotic probability density functions of the arbitrary eigenvalue of the system. The non-asymptotic results are derived for any number of antennas in the system. From the probability density functions, a non-asymptotic and asymptotic expression for the ergodic capacity of the system is derived. We also validated the analytical results by using simulations. The results showed that the non-asymptotic analytical results are in good agreement with the simulations and the asymptotic analytical results provide a good approximation to the exact results even when the system has very few antennas. Also, the results showed that the asymptotic results have much lower complexity compared to the non-asymptotic case.

\section{APPENDIX}

\section{A. Proof of Theorem 1}

Consider the case when $n_{r} \leqslant n_{s}$, then $\boldsymbol{H}_{2}$ has $n_{r}$ non-zero eigenvalues with probability one. The singular value decomposition of $\boldsymbol{H}_{2}$ can be defined as $\boldsymbol{H}_{2}=\boldsymbol{U} \boldsymbol{D}_{2} \boldsymbol{V}^{\dagger}$, where $\boldsymbol{D}_{2}$ is an $n_{r} \times n_{r}$ diagonal matrix with $\left\{\sqrt{\nu_{1}}, \ldots, \sqrt{\nu_{n_{r}}}\right\}$ as the main diagonal elements in decreasing order. Using the singular value decomposition of $\boldsymbol{H}_{2}$, the matrix $\left(\boldsymbol{H}_{2} \boldsymbol{H}_{1}\right)\left(\boldsymbol{H}_{2} \boldsymbol{H}_{1}\right)^{\dagger}$ can be written as $\boldsymbol{U} \boldsymbol{D}_{2} \widetilde{\boldsymbol{H}}_{1} \widetilde{\boldsymbol{H}}_{1}^{\dagger} \boldsymbol{D}_{2} \boldsymbol{U}^{\dagger} \triangleq \boldsymbol{U} \boldsymbol{W}_{0} \boldsymbol{U}^{\dagger}$. The matrix $\boldsymbol{W}_{0}$ is central complex Wishart, as $n_{r} \leqslant n_{s}$, and has $n_{r}$ nonzero eigenvalues defined as $\mu_{1}>\ldots>\mu_{n_{r}}>0$. Then, the unordered conditional density, $f(\boldsymbol{\mu} \mid \boldsymbol{\nu})$ can be given as [15]

$$
\begin{aligned}
f(\boldsymbol{\mu} \mid \boldsymbol{\nu}) & =\frac{1}{n_{r} ! \Gamma_{n_{r}}\left(n_{s}\right) \prod_{k=1}^{n_{r}} \nu_{k}^{n_{s}}\left|\left(\left(-\nu_{i}\right)^{1-j}\right)_{n_{r}}\right|} \\
& \times\left|\left(\sum_{k=1}^{n_{r}} \mu_{k}^{n_{s}-n_{r}+i-1} e^{-\mu_{k} / \nu_{j}}\right)_{n_{r}}\right|,
\end{aligned}
$$

where $\left|\left(\boldsymbol{Q}_{i j}\right)_{l}\right|$ denotes the determinant of the $l \times l$ matrix $\boldsymbol{Q}$ with elements $\boldsymbol{Q}_{i j}$. The unordered non-zero eigenvalue p.d.f. of $\boldsymbol{H}_{2}^{\dagger} \boldsymbol{H}_{2}$ can be found from [16] as

$$
f(\boldsymbol{\nu})=\frac{1}{n_{r} ! \Gamma_{n_{r}}\left(n_{r}\right) \Gamma_{n_{r}}\left(n_{r}\right)} \prod_{k=1}^{n_{r}} e^{-\nu_{k}} \prod_{k<p}^{n_{r}}\left(\nu_{k}-\nu_{p}\right)^{2} .
$$

Now the joint density, $f(\boldsymbol{\mu}, \boldsymbol{\nu})$, can be found using the relation, $f(\boldsymbol{\mu}, \boldsymbol{\nu})=f(\boldsymbol{\nu}) f(\boldsymbol{\mu} \mid \boldsymbol{\nu})$, and integrating $f(\boldsymbol{\mu}, \boldsymbol{\nu})$ over all $\nu_{j}$ we obtain

$$
\begin{aligned}
& f(\boldsymbol{\mu})=\frac{1}{n_{r} ! \Gamma_{n_{r}}\left(n_{r}\right) \Gamma_{n_{r}}\left(n_{r}\right) \Gamma_{n_{r}}\left(n_{s}\right)} \\
& \times\left|\left(\sum_{k=1}^{n_{r}} 2 \mu_{k}^{\left(n_{s}-n_{r}+2 j+i-3\right) / 2} K_{n_{r}-n_{s}+i-1}\left(2 \sqrt{\mu_{k}}\right)\right)_{n_{r}}\right| .
\end{aligned}
$$

The eigenvalue decomposition of $\left(\boldsymbol{H}_{2} \boldsymbol{H}_{1}\right)\left(\boldsymbol{H}_{2} \boldsymbol{H}_{1}\right)^{\dagger}$ can be defined as $\left(\boldsymbol{H}_{2} \boldsymbol{H}_{1}\right)\left(\boldsymbol{H}_{2} \boldsymbol{H}_{1}\right)^{\dagger}=\boldsymbol{V}_{12} \boldsymbol{D}_{12} \boldsymbol{V}_{12}^{\dagger}$, where $\boldsymbol{D}_{12}$ is an $n_{r} \times n_{r}$ diagonal matrix with $\left\{\mu_{1}, \ldots, \mu_{n_{r}}\right\}$ as the main diagonal elements in decreasing order. Hence,

$$
\boldsymbol{W}=\boldsymbol{H}_{3} \boldsymbol{V}_{12} \boldsymbol{D}_{12} \boldsymbol{V}_{12}^{\dagger} \boldsymbol{H}_{3}^{\dagger} \triangleq \widetilde{\boldsymbol{H}}_{3} \boldsymbol{D}_{12} \widetilde{\boldsymbol{H}}_{3}^{\dagger}
$$


Let $\lambda_{1}>\ldots>\lambda_{s_{0}}>0$ be the non-zero eigenvalues of $\boldsymbol{W}$, where $s_{0}=\min \left(n_{d}, n_{r}\right)$, then the conditional unordered eigenvalue p.d.f. $f(\lambda \mid \boldsymbol{\mu})$ can be obtained from [6] as

$f(\lambda \mid \boldsymbol{\mu})=\frac{1}{s_{0} \prod_{k<p}^{n_{r}}\left(\mu_{p}-\mu_{k}\right)} \sum_{k=n_{r}-s+1}^{n_{r}} \frac{\lambda^{n_{d}-n_{r}+k-1}}{\Gamma\left(n_{d}-n_{r}+k\right)}|\boldsymbol{G}|$,

where $\boldsymbol{G}$ is a $n_{r} \times n_{r}$ matrix with entries

$$
\boldsymbol{G}_{i, j}=\left\{\begin{array}{ll}
\mu_{j}^{i-1} & i \neq k \\
\mu_{j}^{n_{r}-n_{d}-1} e^{-\frac{\lambda}{\mu_{j}}} & i=k
\end{array} .\right.
$$

Using the relation $f(\lambda, \boldsymbol{\mu})=f(\boldsymbol{\mu}) f(\lambda \mid \boldsymbol{\mu})$ to find the joint density and integrating $f(\lambda, \boldsymbol{\mu})$ over all $\mu_{j}$ we obtain the result in Theorem 1.

\section{B. Proof of Theorem 2}

When $n_{r}>n_{s}$, the matrix $\boldsymbol{H}_{2}$ also has $n_{r}$ non-zero eigenvalues with probability one. Using the singular value decomposition of $\boldsymbol{H}_{2}$, the matrix $\left(\boldsymbol{H}_{2} \boldsymbol{H}_{1}\right)\left(\boldsymbol{H}_{2} \boldsymbol{H}_{1}\right)^{\dagger}$ can be written as $\boldsymbol{U} \boldsymbol{D}_{2} \widetilde{\boldsymbol{H}}_{1} \widetilde{\boldsymbol{H}}_{1}^{\dagger} \boldsymbol{D}_{2} \boldsymbol{U}^{\dagger} \triangleq \boldsymbol{U} \boldsymbol{W}_{0} \boldsymbol{U}^{\dagger}$. The matrix $\boldsymbol{W}_{0}$ in this case is pseudo Wishart, as $n_{r}>n_{s}$, and has $n_{s}$ nonzero eigenvalues defined as $\mu_{1}>\ldots>\mu_{n_{s}}>0$. Then, the unordered conditional density, $f(\boldsymbol{\mu} \mid \boldsymbol{\nu})$ can be given using results in [17], [18] as

$$
f(\boldsymbol{\mu} \mid \boldsymbol{\nu})=\frac{1}{n_{s} ! \Gamma_{n_{s}}\left(n_{s}\right)\left|\left(\left(\nu_{i}\right)^{j-1}\right)_{n_{r}}\right|}\left|\left(\left(\mu_{i}\right)^{j-1}\right)_{n_{s}}\right||\boldsymbol{E}|,
$$

where $\boldsymbol{E}$ is $n_{r} \times n_{r}$ matrix with elements

$$
\boldsymbol{E}_{i, j}=\left\{\begin{array}{ll}
\nu_{j}^{i-1} & i=1, \ldots, n_{r}-n_{s} \\
\nu_{j}^{n_{r}-n_{s}-1} & i=n_{r}-n_{s}+1, \ldots, n_{r} \\
\times e^{-\mu_{i-n_{r}+n_{s}} / \nu_{j}} &
\end{array} .\right.
$$

The unordered non-zero eigenvalue p.d.f., $f(\boldsymbol{\nu})$, of $\boldsymbol{H}_{2}^{\dagger} \boldsymbol{H}_{2}$ is given in (29). The joint density, $f(\boldsymbol{\mu}, \boldsymbol{\nu})$ can be found again using the relation, $f(\boldsymbol{\mu}, \boldsymbol{\nu})=f(\boldsymbol{\nu}) f(\boldsymbol{\mu} \mid \boldsymbol{\nu})$, and integrating $f(\boldsymbol{\mu}, \boldsymbol{\nu})$ over all $\nu_{j}$ we obtain

$$
f(\boldsymbol{\mu})=\frac{1}{n_{s} ! \Gamma_{n_{s}}\left(n_{s}\right) \Gamma_{n_{r}}\left(n_{r}\right) \Gamma_{n_{r}}\left(n_{r}\right)}\left|\left(\left(\mu_{i}\right)^{j-1}\right)_{n_{s}}\right|\left|\boldsymbol{E}_{2}\right|,
$$

where $\boldsymbol{E}_{2}$ is $n_{r} \times n_{r}$ matrix with elements

$$
\boldsymbol{E}_{2 i, j}=\left\{\begin{array}{c}
(i+j-2) !, \text { for } i=1, \ldots, n_{r}-n_{s} \\
2\left(\mu_{i-n_{r}+n_{s}}\right)\left(n_{r}-n_{s}+j-1\right) / 2 \\
\times K_{n_{r}-n_{s}+j-1}\left(2 \sqrt{\mu_{i-n_{r}+n_{s}}}\right), \\
\text { for } i=n_{r}-n_{s}+1, \ldots, n_{r}
\end{array} .\right.
$$

In this case, the $n_{r} \times n_{r}$ diagonal matrix $\boldsymbol{D}_{12}$ has $\left\{\mu_{1}, \ldots, \mu_{n_{s}}\right\}$ as the non-zero main diagonal elements in decreasing order. Let $\lambda_{1}>\ldots>\lambda_{s_{1}}>0$ be the non-zero eigenvalues of $\boldsymbol{W}$, then the conditional unordered eigenvalue p.d.f. $f(\lambda \mid \boldsymbol{\mu})$ for the case can be obtained from [6] as

$$
f(\lambda \mid \boldsymbol{\mu})=\frac{1}{s_{1} \prod_{k<p}^{n_{s}}\left(\mu_{p}-\mu_{k}\right)} \sum_{k=n_{s}-s_{1}+1}^{n_{s}} \frac{\lambda^{n_{d}-n_{s}+k-1}}{\Gamma\left(n_{d}-n_{s}+k\right)}|\boldsymbol{G}|,
$$

where $G$ is given in (33). Now using (36) and (38) in the relation $f(\lambda, \boldsymbol{\mu})=f(\boldsymbol{\mu}) f(\lambda \mid \boldsymbol{\mu})$ to find the joint density and integrating $f(\lambda, \boldsymbol{\mu})$ over all $\mu_{j}$ we obtain the result in Theorem 2.

\section{REFERENCES}

[1] R. Muller, "A random matrix model of communication via antenna arrays," IEEE Trans. Inf. Theory, vol. 48, no. 9, pp. 2495-2506, Sep. 2002.

[2] D. Chizhik, G. Foschini, M. Gans, and R. Valenzuela, "Keyholes, correlations, and capacities of multielement transmit and receive antennas," IEEE Trans. Wireless Commun., vol. 1, no. 2, pp. 361-368, Apr. 2002.

[3] Y. Karasawa, M. Tsuruta, and T. Taniguchi, "Multi-keyhole model for MIMO radio-relay systems," in Proc. European Conf. on Antennas and Propagation, Edinburgh, UK, Nov. 11-16, 2007, pp. 1-6.

[4] R. U. Nabar, H. Bölcskei, and F. Kneubuhler, "Fading relay channels: Performance limits and space-time signal design," IEEE J. Select. Areas Commun., vol. 22, no. 6, pp. 1099-1109, Aug. 2004.

[5] M. Herdin, "MIMO amplify-and-forward relaying in correlated MIMO channels," in Proc. Int. Conf. on Inform. Commun. and Signal Processing, Bangkok, Thailand, Dec. 6-9, 2005, pp. 796-800.

[6] S. Jin, M. R. McKay, C. Zhong, and K.-K. Wong, "Ergodic capacity analysis of amplify-and-forward MIMO dual-hop systems," in Proc. IEEE Int. Symp. Information Theory (ISIT), Toronto, Canada, July 611, 2008, pp. 1903-1907.

[7] A. Firag, P. Smith, and M. McKay, "Capacity analysis for MIMO twohop amplify-and-forward relaying systems with the source to destination link," in Proc. IEEE Int'l. Conf. on Communications, Dresden, Germany, Jun. 14-18, 2009, pp. 1-6.

[8] H. Bölcskei, R. U. Nabar, O. Oyman, and A. Paulraj, "Capacity scaling laws in MIMO relay networks," IEEE Trans. Wireless Commun., vol. 5 , no. 6 , pp. 1433-1444, June 2006.

[9] J. Wagner, B. Rankov, and A. Wittneben, "On the asymptotic capacity of the Rayleigh fading amplify-and-forward MIMO relay channel," in Proc. IEEE Int. Symp. Information Theory (ISIT), Nice, France, June 24-29, 2007, pp. 2711-2715.

[10] V. Morgenshtern and H. Bölcskei, "Crystallization in large wireless networks," IEEE Trans. Inf. Theory, vol. 53, no. 10, pp. 3319-3349, Oct. 2007.

[11] V. Raghavan and A. Sayeed, "MIMO capacity scaling and saturation in correlated environments," in Proc. IEEE Int'l. Conf. on Communications, vol. 5, Anchorage, Alaska, USA, May 11-15, 2003, pp. 3006-3010.

[12] J. W. Silverstein, "Strong convergence of the empirical distribution of eigenvalues of large dimensional random matrices," Jour. of Multivariate Anal., vol. 55, pp. 331-339, 1995.

[13] R. Muller, "On the asymptotic eigenvalue distribution of concatenated vector-valued fading channels," IEEE Trans. Inf. Theory, vol. 48, no. 7 , pp. 2086-2091, Jul. 2002.

[14] S.-p. Yeh and O. Leveque, "Asymptotic capacity of multi-level amplifyand-forward relay networks," in Proc. IEEE Int. Symp. Information Theory (ISIT), Nice, France, June 24-29, 2007, pp. 1436-1440.

[15] A. Maaref and S. Aïssa, "Eigenvalue distributions of Wishart-type random matrices with application to the performance analysis of MIMO MRC systems," IEEE Trans. Wireless Commun., vol. 6, no. 7, pp. 26782689, July 2007.

[16] T. Ratnarajah, R. Vaillancourt, and M. Alvo, "Complex random matrices and Rayleigh channel capacity," Commun. Inf. Syst., vol. 3, pp. 119-138, 2003.

[17] H. Gao and P. J. Smith, "A determinant representation for the distribution of quadratic forms in complex normal vectors," J. Multivar. Anal., vol. 73, no. 2, pp. 155-165, 2000.

[18] P. J. Smith, S. Roy, and M. Shafi, "Capacity of MIMO systems with semicorrelated flat fading," IEEE Trans. Inf. Theory, vol. 49, pp. 2781$2788,2003$. 